generated with $\mathrm{CD} 45^{+}$cells from FVB-Tac mice and CD $45^{-}$cells from control mice showed normal DETC maturation, as evidenced by coexpression of $\mathrm{V} \gamma 5 \mathrm{~V} \delta 1$ and $\mathrm{CD} 45 \mathrm{RB}$. By contrast, RTOCs generated with FVB-Tac CD $45^{-}$cells and wild-type $\mathrm{CD} 45^{+}$cells contained very few $\mathrm{CD}_{45 \mathrm{RB}}{ }^{\mathrm{hi}} \mathrm{V} \gamma 5 \mathrm{~V} \delta 1^{+}$cells, indicating that the defect in FVB-Tac mice is intrinsic to stromal cells.

Last, the authors confirmed that the delivery of signals through the V $\gamma 5 \mathrm{~V} \delta 1$ TCR by thymic stromal cells was important for the maturation of DETC precursors, as the maturation defect could be rescued by the presence of $17 \mathrm{D} 1$ in the RTOCs.

This study highlights the importance of thymic development for a subpopulation of IELs, as well as an important and often overlooked role for these cells in physiological responses.

Lucy Bird

ORIGINAL RESEARCH PAPER Lewis, J. M. et al Selection of the cutaneous intraepithelial $\gamma \delta^{+}$ $T$ cell repertoire by a thymic stromal determinant. Nature Immunol. 9 July 2006 (doi:10.1038/ni1363)

pro-allergic genes by $\mathrm{CRTH} 2^{+} \mathrm{CD}_{4}{ }^{+}$ T cells, such as those encoding cystatin A and prostaglandin $\mathrm{D}_{2}$ synthase, which might further promote allergic inflammation by central memory $T_{H} 2$ cells. $T$-cell accumulation at the inflammatory sites of atopic dermatitis is associated with high levels of TSLP expression. T cells were found colocalized with activated DCs at these sites, and an examination of these T cells showed that most expressed CRTH2. By contrast, $\mathrm{T}$ cells in lesional skin sections from $\mathrm{T}_{\mathrm{H}} 1$-cellmediated inflammatory diseases, such as psoriasis vulgaris, did not express CRTH2.

So, these data indicate that TSLP-activated DCs might have an important role in the maintenance and reactivation of central memory $\mathrm{T}_{\mathrm{H}} 2$ cells in allergic disease.

Olive Leavy

ORIGINAL RESEARCH PAPER Wang, Y-H. et al. Maintenance and polarization of human $\mathrm{T}_{\mathrm{H}} 2$ central memory $T$ cells by thymic stromal lymphopoietin-activated dendritic cells. Immunity 24, 827-838(2006)

\title{
Defective genes at checkpoints
}

Systemic lupus erythematosus (SLE) is an autoimmune disease that is characterized by the presence of antibodies specific for self nucleic acids or proteins associated with these nucleic acids. For several decades, researchers have been trying to pin down the precise means by which tolerance to self molecules breaks down in individuals with SLE and to identify the various genes and proteins that underlie this disorder. In both mice and humans, SLE seems to be polygenic, and it is known to be associated with more than 12 genetic loci. Now, two papers published in Science explain the contribution of several loci to SLE in mice; together with previous findings, these results provide a potential explanation for the pathogenesis of SLE.

During B-cell development in the bone marrow, a diverse repertoire of $\mathrm{B}$-cell receptors (BCRs) is generated through the random rearrangement of immunoglobulin gene segments. Many of these rearrangements result in BCRs that bind self antigens. However, such autoreactive $B$ cells do not usually reach the periphery, because intracellular signalling through a self-reactive BCR results in cell death, anergy or receptor editing (in which new rearrangements generate a modified BCR).

To study how these tolerance mechanisms might break down and lead to SLE, Kumar et al. investigated the Sle1 locus, which is known to be an SLE-susceptibility locus in mice and has an orthologue in humans. The authors show that inherited differences in one of the genes encoded in this region, Ly108, result in variations in BCR signalling: the presence of $S l e 1 b^{z}$, an allele that is present in a strain of SLE-prone mice, reduces the ability of self-reactive $B$ cells to undergo anergy or receptor editing and therefore increases the number of self-reactive B cells in the periphery.

Pisitkun et al. studied another SLE-susceptibility locus in mice, the well-known Y-chromosomelinked autoimmune accelerator (Yaa) locus. They found that Yaa is a result of duplication of a segment of the $X$ chromosome that has been transposed to the $Y$ chromosome and contains the Toll-like receptor 7 (Tlr7) gene. Consequently, the $B$ cells of Yaa mice express double the amount of TLR7 and are twice as sensitive to TLR7 ligands as are $B$ cells from wild-type mice. Because TLR7 is present in the endosome, it would normally come into contact only with microbial nucleic acids, but mammalian nucleic acids can bind TLR7 if they are delivered to the endosome through internalization of cell-surface BCR. In addition, the phenotypic effects of $\mathrm{Yaa}$

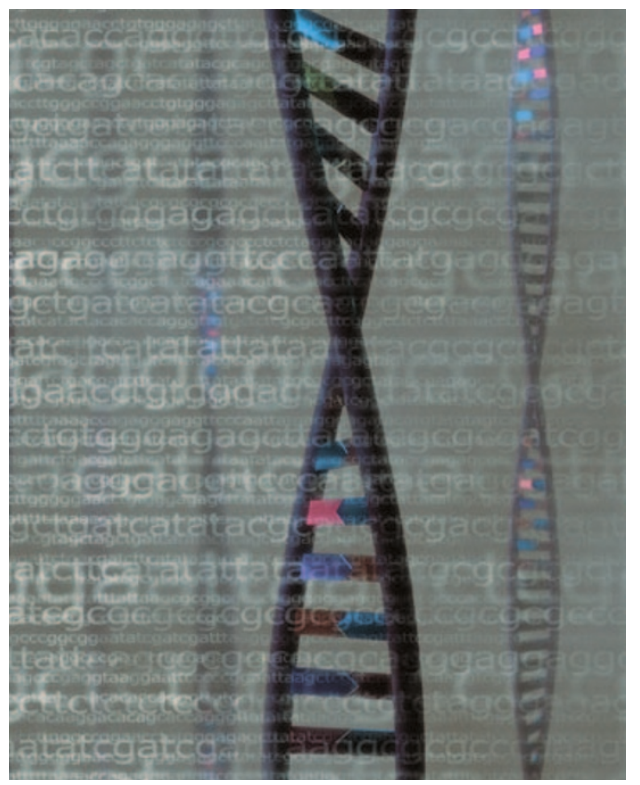

are known to be modified through interactions with other genes. The authors show that the presence of an SLE-associated allele of the inhibitory receptor FcyRllb, together with Yaa, increases the severity and incidence of SLE symptoms.

Because of the polygenic nature of SLE, these findings, taken together with those from previous studies, indicate that self-tolerance is likely to be broken by a succession of defective checkpoints (see Further reading). First, B cells might have a defect in the induction of anergy and receptor editing (as exemplified by Sle $1 b^{z}$ ), thereby increasing the pool of self-reactive $B$ cells in the periphery. Second, peripheral B cells might have a defect in the inhibitory signalling that normally opposes BCR signalling (as in the case of loss of Fc $\gamma R$ Illb function). Last, peripheral B cells might have a defect in discrimination between self and microbial nucleic acids by TLRs (as in the case of Yaa mice), thereby inducing the proliferation of self-reactive B cells. Each successive defect therefore removes any redundant controls in the system and compounds the problem, allowing T-cell-independent proliferation and autoantibody production by $B$ cells.

Davina Dadley-Moore

ORIGINAL RESEARCH PAPERS Kumar, K. R. et al. Regulation of $B$ cell tolerance by the lupus susceptibility gene $L y 108$. Science 312 1665-1669 (2006) | Pisitkun, P. et al. Autoreactive B cell responses to RNA-related antigens due to TLR7 gene duplication. Science 312, 1669-1672 (2006)

FURTHER READING Goodnow, C. C. Discriminating microbe from self suffers a double Toll. Science 312, 1606-1608 (2006) 